\title{
Eosinophilic crystalline pneumonia, an age-related lesion in mice
}

\author{
Jenna J Klug ${ }^{\mathrm{a},}$, Jessica M Snyder $^{\mathrm{a}}$ \\ ${ }^{a}$ Department of Comparative Medicine, School of Medicine, University of Washington, Seattle, WA, USA.
}

\begin{abstract}
Eosinophilic crystalline pneumonia (ECP), also known as acidophilic macrophage pneumonia (AMP), is a common intrapulmonary lesion that increases in prevalence with age in mice, especially those on a C57BL/6 and 129Sv background. Gross changes may be evident in severe cases as lobar to diffuse red to brown foci throughout the lungs, which fail to collapse. Definitive diagnosis is by histopathology, which shows the accumulation of brightly eosinophilic crystals within macrophages or free within lumens of alveolar spaces and conducting airways. Granulocytes, multinucleated giant cells, and epithelial hyalinosis may also be present in affected areas of the lung. The disease may represent a cause of morbidity and mortality when other disease processes interfere with clearance, leading to the accumulation of crystals and crystal laden macrophages in airways, resulting in dyspnea. Other anatomic locations may be affected by epithelial hyalinosis and/or crystals as part of the syndrome, including respiratory tract, stomach, gall bladder, bile duct, and pancreatic duct.

Keywords: Epithelial hyalinosis, crystals, acidophilic macrophage pneumonia (AMP), eosinophilic crystalline pneumonia (ECP)
\end{abstract}

Eosinophilic crystalline pneumonia (ECP), also known as acidophilic macrophage pneumonia (AMP), is a common pulmonary lesion that increases in incidence with age in mice [1]. It occurs across most laboratory strains and wild mice, although has a higher prevalence in $\mathrm{C} 57 \mathrm{BL} / 6$, 129Sv, Swiss, Ptpn6me motheaten mice, severe combined immunodeficiency (SCID), and various types of genetically engineered mice on a C57BL/6 or 129 background $[1,2]$. Gross changes range from multifocal parenchymal infiltrates to lobar to diffuse areas of red and tan discolorations in the lungs, which fail to collapse upon opening the thoracic cavity [1]. Definitive diagnosis is made by histopathology and characterized by an intrapulmonary accumulation of brightly eosinophilic acicellular (needleshaped) to rectangular crystals [1, 3, 4]. Crystals may be present extracellularly within alveolar spaces and conducting airways (Figure 1, Figure 2C), or within the cytoplasm of macrophages and multinucleated giant cells (Figure 2A, B). Affected areas of lung may also contain granulocytes and epithelial hyalinosis $[2,4]$.

\footnotetext{
* Corresponding author: Jenna J Klug
}

Mailing address: Department of Comparative Medicine, School of Medicine, University of Washington, Seattle, WA, USA.

Email: jklug13@uw.edu

Received: 18 November 2020 / Accepted: 15 December 2020
ECP can occur spontaneously or concurrently with other lung pathology, such as neoplastic, hyperplastic, infectious, hypersensitivity, and lymphoproliferative diseases [1]. In aging mice, the condition may represent a cause of morbidity and mortality when found in association with any disease process that impairs normal clearance of alveolar exudate, causing large numbers of crystal-laden macrophages to accumulate in air spaces, leading to respiratory distress and death [2]. The crystals are composed of chitinase-3-like-3 (CHI3L3) protein (formerly known

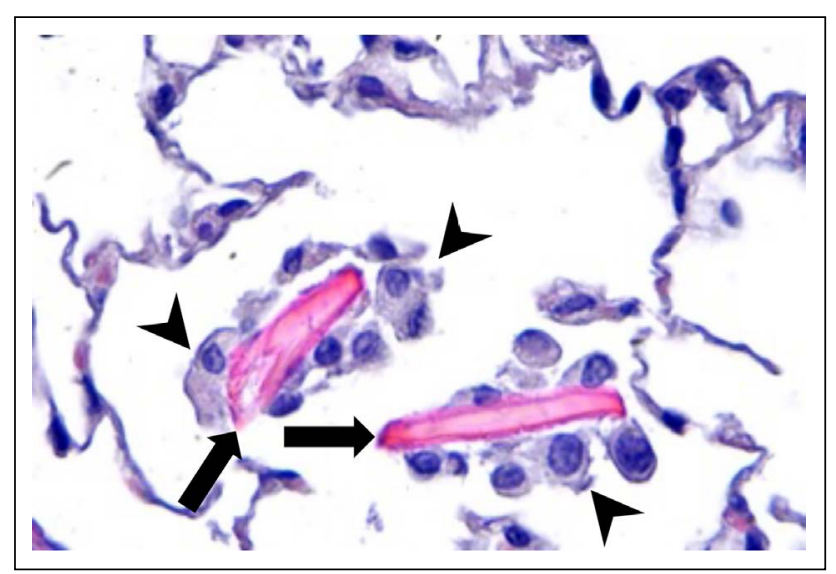

Figure 1. Eosinophilic crystalline pneumonia in a 28-month-old male C57BL/6 mouse. ECP crystals (arrows) in an alveolar sac of the lung. The crystals are large, eosinophilic, rectangular, extracellular, and associated with macrophage infiltrates (arrowheads), 400x, HE. 

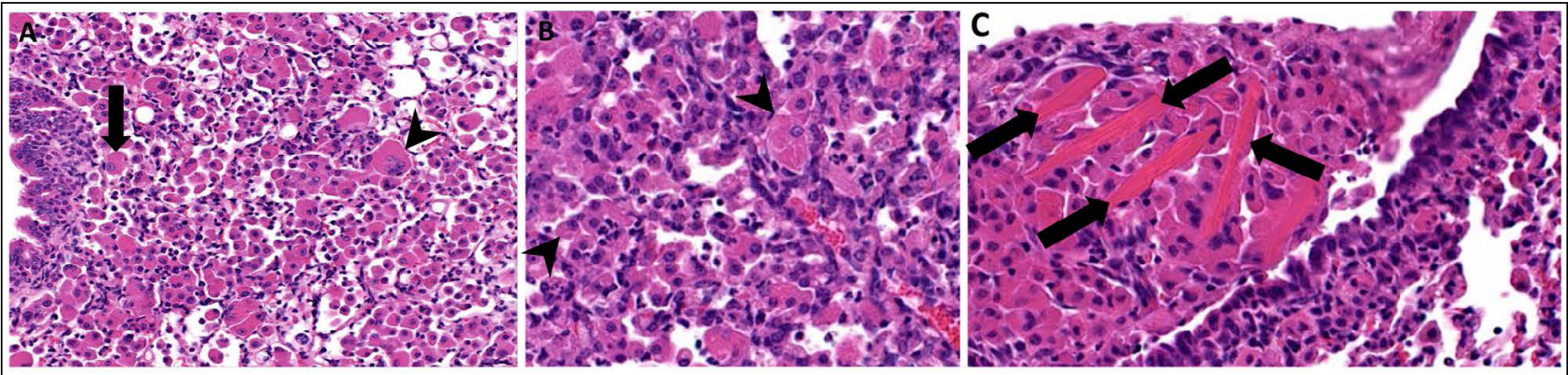

Figure 2. Eosinophilic crystalline pneumonia in a 16-month-old female genetically engineered mouse associated with pulmonary adenocarcinoma. (A) Lung moderate to severely affected by ECP. Large numbers of macrophages are present within alveolar spaces, with some multinucleated giant cell formation. Intracellular eosinophilic crystals are present within some macrophages (arrow) and multinucleated giant cells (arrowhead), 200x, HE. (B) Higher magnification of ECP demonstrating small, needle-shaped, eosinophilic crystals within the cytoplasm of macrophages (arrowhead), 400x, HE. (C) Higher magnification of ECP demonstrating large, rectangular extracellular eosinophilic crystals, (arrows) 400x, HE.

as YM1) and contain iron, alpha-1 antitrypsin, immunoglobulin, and granulocyte breakdown products $[2,5]$. Morphologically, they are similar to Charcot-Leyen crystals, which are present in humans and nonhuman primates with eosinophil-rich diseases such as asthma and helminth infestations [1]. Although the lesions in the lungs are the most overt manifestation of this condition, hyalinosis may also occur at other anatomical locations as part of the syndrome in predisposed mice, including epithelium of olfactory, nasal respiratory, middle ear, trachea, lung, stomach, gall bladder, bile duct and pancreatic ducts $[2,5,6]$. In addition, extracellular crystals may also be present in the glands of these tissues [3].

\section{Declarations}

Authors' contributions: Jenna Klug wrote the first draft of the manuscript. Jessica Synder contributed to editing and additions.

Financial support and sponsorship: Supported by NIA grant R01 AG05-7381 (Warren Ladiges, PI).

Conflict of interest: The authors declare no conflict of interest.

Consent for publication: The authors consent to the pub- lication of this manuscript.

\section{References}

1. Hoenerhoff M J, Starost M F, Ward J M. Eosinophilic crystalline pneumonia as a major cause of death in 129S4/ SvJae mice. Veterinary Pathology, 2006, 43(5): 682-688.

2. Barthold SW, Griffey S M, D H. Pathology of laboratory rodents and rabbits. 4th ed. New York: Wiley 2016: 94-95.

3. Cesta M F, Dixon D, Herbert R A , et al. Lung-Crystals. National Toxicology Program Nonneoplastic Lesion Atlas. 2015. Abstract: https://ntp.niehs.nih.gov/nnl/respiratory/lung/crystal/index.htm

4. Pettan-Brewer C, M. Treuting P M. Practical pathology of aging mice. Pathobiology of Aging \& Age-related Diseases, 2011, 1(1): 7202.

5. Ward J M, Yoon M, Anver M R, et al. Hyalinosis and Ym1/ Ym2 gene expression in the stomach and respiratory tract of 129S4/SvJae and wild-type and CYP1A2-null B6, 129 mice. The American journal of pathology, 2001, 158(1): 323-332.

6. Thoolen B, Maronpot R R, Harada T, et al. Proliferative and nonproliferative lesions of the rat and mouse hepatobiliary system. Toxicologic pathology, 2010, 38(7. suppl): 5S-81S.

Cite this article as: Klug $\mathrm{J} \mathrm{J}$, Snyder J M. Eosinophilic crystalline pneumonia, an age-related lesion in mice[J]. Aging Pathobiology and Therapeutics, 2020, 2(4): 232-233. 